\title{
Erratum to: Evidence-based clinical practice guidelines for polycystic kidney disease 2014
}

\author{
Shigeo Horie ${ }^{1} \cdot$ Toshio Mochizuki $^{2} \cdot$ Satoru Muto $^{3} \cdot$ Kazushige Hanaoka $^{4} \cdot$ \\ Yoshimitsu Fukushima $^{5}$ - Ichiei Narita ${ }^{6}$ Kikuo Nutahara ${ }^{7} \cdot$ Ken Tsuchiya $^{2}$. \\ Kazuhiko Tsuruya $^{8} \cdot$ Koichi Kamura $^{9}$ - Saori Nishio ${ }^{10}$ - Tatsuya Suwabe ${ }^{11}$. \\ Yoshifumi Ubara $^{11}$ - Eiji Ishimura ${ }^{12}$ - Koichi Nakanishi ${ }^{13} \cdot$ Keiichi Furukawa $^{14}$. $^{2}$ \\ Kenjiro Kimura $^{15} \cdot$ Seiichi Matsuo ${ }^{16}$
}

Published online: 29 June 2016

(C) Japanese Society of Nephrology 2016

\section{Erratum to: Clin Exp Nephrol \\ DOI 10.1007/s10157-015-1219-7}

Unfortunately in the original version of the above article, CQ 13 was processed incorrectly.

CQ 13 has been corrected with this erratum.

CQ 13. Should ADPKD patients with ESRD undergo hepatic transarterial embolization to reduce hepatomegaly?

Recommended Grade: C1

Hepatic transarterial embolization in ADPKD patients with ESRD is effective in reducing hepatomegaly and is therefore recommended.
The online version of the original article can be found under doi:10.1007/s10157-015-1219-7.

Shigeo Horie

shorie@juntendo.ac.jp

Juntendo University Graduate School of Medicine, Tokyo, Japan

2 Tokyo Women's Medical University, Tokyo, Japan

3 Teikyo University School of Medicine, Tokyo, Japan

4 Department of Internal Medicine, Jikei University School of Medicine, Tokyo, Japan

5 Shinshu University School of Medicine, Matsumoto, Japan

6 Niigata University Graduate School of Medical and Dental Sciences, Niigata, Japan

7 Kyorin University, Tokyo, Japan

8 Graduate School of Medical Sciences, Kyushu University, Fukuoka, Japan
9 National Hospital Organization Chiba-East Hospital, Chiba, Japan

10 Hokkaido University Graduate School of Medicine, Sapporo, Japan

11 Toranomon Hospital, Tokyo, Japan

12 Osaka City University Graduate School of Medicine, Osaka, Japan

13 Wakayama Medical University, Wakayama, Japan

14 St. Luke's International Hospital, Tokyo, Japan

15 St. Marianna University School of Medicine, Kawasaki, Japan

16 Nagoya University Graduate School of Medicine, Nagoya, Japan 